\title{
Inverse Problem of Failure Mechanics for a Drawing Die Strengthened with a Holder
}

\author{
Vagif M. Mirsalimov \\ Azerbaijan Technical University \\ Baku, Azerbaijan \\ E-mail: mir-vagif@mail.ru
}

\section{Farid E. Veliyev}

Institute of Mathematics and Mechanics of NAS of Azerbaijan

Baku, Azerbaijan

E-mail: iske@mail.ru

Abstract: A plane problem of failure mechanics is considered for concentrically integrated cylinders. It is assumed that the drawing die (internal cylinder) is negative allowance strengthened by means of external cylinder (holder), and near the surface of the drawing die there are $N$ arbitrarily located rectilinear cracks of length $2 l_{k}(k=1,2, \ldots, N)$. Theoretical analysis on definition the negative allowance providing minimization of failure parameters (stress intensity factors) of drawing die was carried out on minimax criterion. A simplified method for minimization the failure parameters of a hard alloy drawing die was separately considered.

Keywords: hard-alloy drawing die; reinforcing cylinder; negative allowance; cracks; stress intensity factors; minimization of drawing die failure parameters

\section{Introduction}

Experience shows [1] the great reliability and durability of multicomponent constructions compared to homogeneous ones. At present, sandwich constructions are widely used in industry and engineering. While designing high pressure apparatus, a circuit of negative allowance connected multicomponent ring under internal pressure is often used. A similar circuit is implemented in draw-making while drawing the wires and rods of annular cross section. The drawing is a process when a wire, a rod or a pipe is given a draft through the hole of a special instrument (drawing die) that has some less section than the initial work piece. 
The drawing dies are manufactured from hard alloys, industrial diamonds (to make thin rods) or tool steel (to draw rods and large section pipes). The hard alloys and diamond are embedded so that it could freely go in a draw hole and go out from the opposite side. The end is caught by a tractive mechanism [2] of a drawbench that gives the rod a draft through a drawing block and subjects it to deformation, i.e. to reduction and drawing.

The experience of the drawing industry shows that [2] the failure of hard alloy drawing dies with reinforcing rings (holder) occurs because of crack propagation arising on the boundary of the working and calibrating zones of the drawing die. In this connection, at the stage design of new constructions of drawing dies, it is necessary to perform limit analysis of the drawing die in order to determine that the would-be initial cracks arranged unfavorably will not grow to disastrous sizes and cause failure in the course of rated life. The size of the initial minimal crack should be considered as a design characteristic of the material.

At the current stage of development of engineering, the optimal design of the machine parts provided in order to increase their serviceability is of great importance. Therefore, the optimal design of composite (multicomponent) constructions increases in importance. An increase in the drawing die's serviceability may be substantially controlled by using design-technological methods, in particular by geometry negative allowance of the connection of a drawing die and a holder. The solution of a problem of mechanics on the determination of such negative allowance of a drawing die and reinforcing ring under which the stress field created by this tension could slow down the crack propagation in the drawing die, which is of particular interest.

\section{Formulation of the Problem}

Let us consider a stress-strain state in a hard alloy drawing die reinforced with a holder under the action of loads normal and tangential to the inner contour. It is accepted that the inner contour of the drawing die orifice is close to annular one. As is known, the real surface of the tool is never absolutely smooth and always has micro or macroscopic irregularities of a technological character. In spite of exceptionally small sizes of the unevenness that generate roughness, it has an essential effect on various operational properties of tools [3-6].

It is assumed that a hard alloy drawing die is negative allowance reinforced with the help of an annular ring (holder) made of mean carbon steel. The allowance function is not known beforehand and should be defined. Let a negative allowance reinforced elastic drawing die with an outer cylinder (ring) have $N$ rectilinear cracks of length $2 l_{k}(k=1,2, \ldots, N)$. At the center of the cracks, locate the origin of local coordinate systems $x_{k} O_{k} y_{k}$ whose axis $x_{k}$ coincides with the lines of cracks 
and makes the angle $\alpha_{k}$ with the axis $x$ (Fig. 1). It is assumed that the cracks' lips are free from external loads. Refer the two-component ring to the polar coordinate $r \theta$ system of having chosen the origin at the center of concentric circles $L_{0}, L, L_{1}$ with radii $R_{0}, R, R_{1}$ (Fig. 1), respectively.

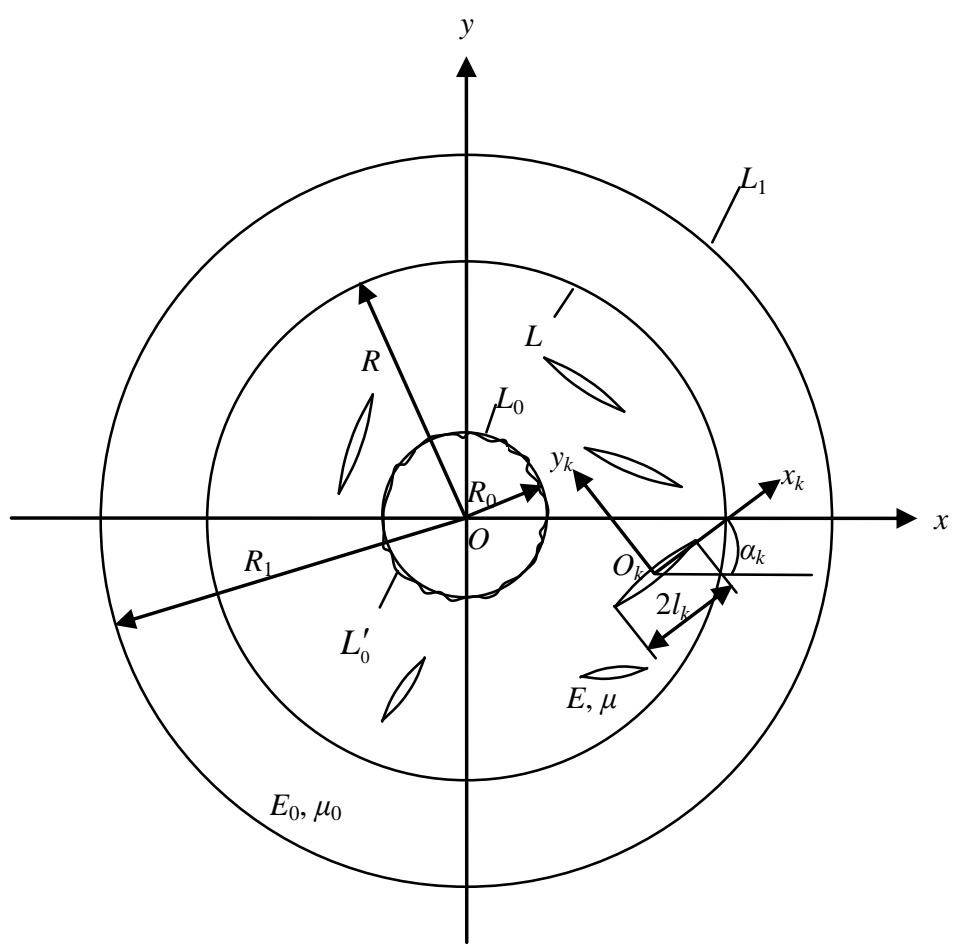

Figure 1

Calculation scheme of inverse problem of failure mechanics for a drawing die with reinforcing cylinder

Consider some realization of the rough inner surface of the drawing die. We will assume that the plane stress state condition is fulfilled. In the area occupied by the two-component ring (drawing die and holder), the stress tensor components $\sigma_{r}, \sigma_{\theta}$, $\sigma_{r \theta}$ should satisfy the differential equations of plane theory of elasticity [7].

Denote by $E, \mu$ and $E_{0}, \mu_{0}$ the Young modulus and Poisson ratio of the drawing die and reinforcing ring, respectively. The boundary of the inner contour $L_{0}$ is presented in the form:

$r=\rho(\theta)=R_{0}+\delta(\theta)$ 
Let the outer contour $L_{1}$ be free from loads. The boundary conditions of the considered problem are of the form:

$$
\begin{aligned}
& \sigma_{n}=-p, \quad \tau_{n t}=-f p \text { for } r=\rho(\theta) \\
& \sigma_{r}=0, \quad \tau_{r \theta}=0 \text { for } r=R_{1} \\
& \sigma_{r}^{0}-i r_{r \theta}^{0}=\sigma_{r}^{d}-i r_{r \theta}^{d}, v_{r}^{0}-i v_{r \theta}^{0}=v_{r}^{d}-i v_{r \theta}^{d}-g(\theta) \text { for } r=R \\
& \sigma_{n}^{d}=0, \quad \tau_{n t}^{d}=0 \quad \text { on the crack's faces }
\end{aligned}
$$

Here $v_{r}, v_{\theta}$ are radial and tangential constituents of the vectors of the displacement points of the contour $L ; g(\theta)$ is the desired allowance function; $f$ is the friction factor of the "drawing die-wire rod" pair; $i=\sqrt{-1} ; p$ is the pressure on the inner surface of the drawing die.

The temperature of the surface layers of the drawing die increases under drawing under the action of contact friction. By drawing on the inner surface of the drawing die, on the area of contact friction with wire (wire rod), there acts a surface heat source heat caused by the outer friction. Tangential forces $\tau=f p$ promote the release of heat in the contact area of the tool and the wire rod in the drawing process. The general amount of heat in a time unit is proportional to the power of the friction forces, and the amount of the heat released at the point in the contact zone with coordinate $\theta$ will be equal to

$$
Q(\theta)=V f p,
$$

where $V$ is the mean displacement velocity of the wire rod with respect to the drawing die (drawing velocity).

The total amount of heat $Q(\theta)$ will be consumed as follows: heat flow in the drawing die $Q_{d}(\theta)$ and similar heat flow $Q_{1}(\theta)$ for increasing the wire rod heat.

In the case of steady heat exchange, the definition of the temperature field in the drawing die and annular holder may be reduced to the solution of boundary value problem of heat-conductivity

in the drawing die $\Delta T=0$

in the reinforcing ring $\Delta T_{0}(r, \theta)=0$

for $r=R: T=T_{0}, \lambda \frac{\partial T}{\partial n}=\lambda_{0} \frac{\partial T_{0}}{\partial n}$

for $r=\rho(\theta): \lambda \frac{\partial T}{\partial n}=-Q_{d}(\theta)$ on the contact area

for $r=R_{1}: \lambda_{0} \frac{\partial T_{0}}{\partial r}+\alpha_{2}\left(T_{0}-T_{2}\right)=0$ 
Here $T$ is the temperature in an elastic isotropic drawing die; $T_{0}$ is the temperature in the reinforcing cylinder; $\lambda, \lambda_{0}$ are the thermal conductivity coefficients of the drawing die and holder, respectively; $\Delta$ is Laplace's operator; $T_{2}$ is the temperature of environment on the external surface of the holder; $\alpha_{2}$ is the heat exchange from the outer cylindrical surface of the holder with external medium; $Q_{d}(\theta)=\alpha_{d} f p V$ is the intensity of the surface heat source for a drawing die; $\alpha_{d}$ is a coefficient of heat flow separation for a drawing die.

For finding the allowance function, the statement of the problem should be complemented by a condition (criterion) that allows us to determine the desired negative allowance.

According to the Irvin-Orovan theory [8] of quasibrittle fracture, the stress intensity factor is a parameter characterizing the stress state in the vicinity of the crack end. Consequently, the maximal value quantity of the stress intensity coefficient near the crack tip is responsible for the failure of the drawing die's material. Investigating the basic failure parameters and the influence of allowance of the drawing die's junction and reinforcing ring, the material properties and other factors on them, we can substantially control the failure by designtechnological methods, in particular by varying the negative allowance (the function $g(\theta)$ ). Further, we accept minimization of quantity of maximal stress intensity factors on the vicinity of the crack tips in the drawing die. The minimization of the maximal value of the stress intensity coefficient will promote an increase in the serviceability of the drawing die of the drawing tool.

Thus, it is required to determine the junction negative allowance $g(\theta)$ such that the stress field created by it in the loading process prevents the crack from propagating.

Not losing the generality of the stated problem, it is accepted that the desired allowance function $g(\theta)$ may be represented as a Fourier series. Consequently, the coefficients $A_{k}^{d}=\alpha_{k}+i \beta_{k}$ in the expansion of the desired allowance function should be managed so that the minimization of the maximal stress intensity factors are provided. This additional condition allows to determine the desired function $g(\theta)$.

\section{The Case of a Single Crack}

In order to solve the stated inverse problem, it is necessary to solve a problem of failure mechanics for the "drawing die and reinforcing holder" pair. Represent the boundary of the internal contour $L_{0}^{1}$ of the drawing die in the form:

$r=\rho(\theta)=R_{0}+\varepsilon H(\theta)$ 


$$
H(\theta)=\sum_{k=0}^{n}\left(a_{k}^{0} \cos k \theta+b_{k}^{0} \sin k \theta\right)
$$

where $\varepsilon=R_{\max } / R_{0}$ is a small parameter, $R_{\max }$ is the greatest height of the bulge of irregularity of the surface friction, and $H(\theta)$ is a function independent of a low parameter.

Using a profilometer, the measurements have been made for a treated surface of the drawing die, and the approximate values Fourier coefficients for the function $H(\theta)$ describing each inner profile of the treated drawing die surface have been calculated for the function $H(\theta)$.

We look for temperatures, the stress tensor components and the displacements in the drawing die and holder in the form of expansions in small parameter $\varepsilon$ :

$$
\begin{aligned}
& T=T^{(0)}+\varepsilon T^{(1)}+\ldots, T_{0}=T_{0}^{(0)}+\varepsilon T_{0}^{(1)}+\ldots, \\
& \sigma_{r}=\sigma_{r}^{(0)}+\varepsilon \sigma_{r}^{(1)}+\ldots, \quad \sigma_{\theta}=\sigma_{\theta}^{(0)}+\varepsilon \sigma_{\theta}^{(1)}+\ldots, \quad \tau_{r \theta}=\tau_{r \theta}^{(0)}+\varepsilon \tau_{r \theta}^{(1)}+\ldots, \\
& v_{r}=v_{r}^{(0)}+\varepsilon v_{r}^{(1)}+\ldots, \quad v_{\theta}=v_{\theta}^{(0)}+\varepsilon v_{\theta}^{(1)}+\ldots, \quad g(\theta)=g^{(0)}(\theta)+\varepsilon g^{(1)}(\theta)+\ldots
\end{aligned}
$$

where the terms with $\varepsilon$ of higher order are neglected for simplification. Here $T^{(0)}, T_{0}^{(0)}$ are zero approximation temperatures; $T^{(1)}, T_{0}^{(1)}$ are first approximation temperatures, respectively; $\sigma_{r}^{(0)}, \sigma_{\theta}^{(0)}, \tau_{r \theta}^{(0)}$ are zero approximation stresses; $\sigma_{r}^{(1)}, \sigma_{\theta}^{(1)}$ and $\tau_{r \theta}^{(1)}$ are first approximation stresses; $v_{r}^{(0)}, v_{\theta}^{(0)}$ are radial and tangential displacements at a zero approximation; and $v_{r}^{(1)}, v_{\theta}^{(1)}$ are first approximation displacements. Each of the above approximations satisfies the system of differential equations of the plane theory of elasticity [7]. Expanding in series the expressions for temperature, stresses and displacements in the vicinity $r=R_{0}$ we obtain the values of constituents of temperature, stress sensor and displacement components for $r=\rho(\theta)$.

Using the perturbations method, with regard to what has been said, we arrive at the sequence of boundary conditions for the boundary value problems of fracture mechanics for a drawing die and reinforcing cylinder

at a zero approximation for $r=R_{0} \quad \lambda \frac{\partial t^{(0)}}{\partial r}=-Q$

for $r=R t^{(0)}=t_{0}^{(0)} ; \lambda \frac{\partial t^{(0)}}{\partial r}=\lambda_{0} \frac{\partial t_{0}^{(0)}}{\partial r}$

for $r=R_{1} \quad \lambda_{0} \frac{\partial t_{0}^{(0)}}{\partial r}+\lambda_{2} t_{0}^{(0)}=0$

for $r=R_{0} \quad \sigma_{r}^{d(0)}=-p ; \tau_{r \theta}^{d(0)}=-f p$ 
for $r=R \quad \sigma_{r}^{0(0)}-i \tau_{r \theta}^{0(0)}=\sigma_{r}^{d(0)}-i \tau_{r \theta}^{d(0)}$

$v_{r}^{0(0)}-i v_{\theta}^{0(0)}=v_{r}^{d(0)}-i v_{\theta}^{d(0)}-g^{(0)}(\theta)$

for $r=R_{1} \quad \sigma_{r}^{0(0)}=0 ; \quad \tau_{r \theta}^{0(0)}=0$

on the crack faces $\sigma_{y_{1}}^{d(0)}=0 ; \tau_{x_{1} y_{1}}^{d(0)}=0$ for $y_{1}=0,\left|x_{1}\right| \leq l_{1}$

in the first approximation for $r=R_{0} \frac{\partial t^{(1)}}{\partial r}=-\frac{\partial^{2} t^{(0)}}{\partial r^{2}} H(\theta)$,

for $r=R t^{(1)}=t_{0}^{(1)} ; \lambda \frac{\partial t^{(1)}}{\partial r}=\lambda \frac{\partial^{2} t_{0}^{(1)}}{\partial r^{2}}$

for $r=R_{1} \quad \lambda_{0} \frac{\partial t_{0}^{(0)}}{\partial r}+\alpha_{2} t_{0}^{(1)}=0$,

for $r=R_{0} \quad \sigma_{r}^{d(1)}=N_{0} ; \tau_{r \theta}^{d(1)}=T_{0}$

for $r=R \quad \sigma_{r}^{0(1)}-i \tau_{r \theta}^{0(1)}=\sigma_{r}^{d(1)}-i \tau_{r \theta}^{d(1)}$,

$v_{r}^{0(1)}-i v_{\theta}^{0(1)}=v_{r}^{d(1)}-i v_{\theta}^{d(1)}-g^{(1)}(\theta)$

for $r=R_{1} \quad \sigma_{r}^{0(1)}=0 ; \tau_{r \theta}^{0(1)}=0$

on the crack faces $\sigma_{y_{1}}^{d(1)}=0 ; \tau_{x_{1} y_{1}}^{d(1)}=0$ for $y_{1}=0,\left|x_{1}\right| \leq l_{1}$

Here $t=T-T_{c} ; t_{0}=T_{0}-T_{c}$ are excessive temperatures; $T_{\mathrm{c}}$ is temperature of environment

$$
\begin{aligned}
& \text { for } r=R_{0} \quad N_{0}=-H(\theta) \frac{\partial \sigma_{r}^{d(0)}}{\partial r}+2 \tau_{r \theta}^{d(0)} \frac{1}{R_{0}} \frac{d H(\theta)}{d \theta} \\
& T_{0}=\left(\sigma_{\theta}^{d(0)}-\sigma_{r}^{d(0)}\right) \frac{1}{R_{0}} \frac{d H(\theta)}{d \theta}-H(\theta) \frac{\partial \tau_{r \theta}^{d(0)}}{\partial r} .
\end{aligned}
$$

At each approximation, the solution of the boundary value problem of heat conductivity theory is sought by the method of separation of variables. We find temperatures $t$ for a drawing die and $t_{0}$ for a reinforcing cylinder in the form

$t^{(0)}=C_{1}^{0}+C_{2}^{0} \ln \rho, \rho=r / R$,

$t_{0}^{(0)}=C_{3}^{0}+C_{4}^{0} \ln \rho_{1}, \rho_{1}=r / R_{1}$,

$t^{(1)}=C_{1}+C_{2} \ln \rho+\sum_{k=1}^{\infty}\left(C_{1}^{(k)} \rho^{k}+C_{2}^{(k)} \rho^{-k}\right) \cos k \theta+\sum_{k=1}^{\infty}\left(A_{1}^{(k)} \rho^{k}+A_{2}^{(k)} \rho^{-k}\right) \sin k \theta$, 
$t_{0}^{(1)}=C_{3}+C_{4} \ln \rho_{1}+\sum_{k=1}^{\infty}\left(C_{3}^{(k)} \rho_{1}^{k}+C_{4}^{(k)} \rho_{1}^{-k}\right) \cos k \theta+\sum_{k=1}^{\infty}\left(A_{3}^{(k)} \rho_{1}^{k}+A_{4}^{(k)} \rho_{1}^{-k}\right) \sin k \theta$.

The constants $C_{1}^{0}, C_{2}^{0}, C_{3}^{0}, C_{4}^{0}, C_{1}, C_{2}, C_{3}, C_{4}, C_{1}^{(k)}, C_{2}^{(k)}, C_{3}^{(k)}, C_{4}^{(k)}, A_{1}^{(k)}, A_{2}^{(k)}, A_{3}^{(k)}, A_{4}^{(k)}$ are determined from the boundary conditions of the thermal conductivity theory problem (8), (10). Because of their length, the corresponding formulae are not presented here. To solve the thermoelasticity problem, we will use the thermoelastic displacement potential [9].

In the considered problem, the thermoelastic displacement potential for a drawing die $F$ and reinforcing cylinder $F_{0}$ is determined at each approximation by the solution of the following differential equations

$$
\Delta F^{(j)}=\frac{1+\mu}{1-\mu} \alpha t^{(j)}, \Delta F_{0}^{(j)}=\frac{1+\mu_{0}}{1-\mu_{0}} \alpha_{0} t_{0}^{(j)} \quad(j=0,1)
$$

Here $\alpha, \alpha_{0}$ are the coefficients of linear temperature expansion for a drawing die and reinforcing holder, respectively, and $\mu, \mu_{0}$ are the Poisson ratio of a drawing die and reinforcing cylinder material. We will seek a solution of equations (14) in the form:

$$
F=\sum_{n=0}^{\infty}\left(f_{n} \cos n \theta+f_{n}^{*} \sin n \theta\right), \quad F_{0}=\sum_{n=0}^{\infty}\left(f_{n o} \cos n \theta+f_{n o}^{*} \sin n \theta\right)
$$

At each approximation, for the functions $f_{n}(r), f_{n}^{*}(r), f_{n o}(r), f_{n o}^{*}(r)$, we obtain ordinary differential equations whose solutions are found by the method of variation of the constants. After determining the thermoelastic displacement potentials for a drawing die and reinforcing cylinder using well known formulae [9], we calculate the stresses $\bar{\sigma}_{r}^{d(j)}, \bar{\sigma}_{\theta}^{d(j)}, \bar{\sigma}_{r \theta}^{d(j)}$ and displacements $\bar{v}_{r}^{d(j)}, \bar{v}_{\theta}^{d(j)}$ for a drawing die, and also $\bar{\sigma}_{r}^{o(j)}, \bar{\sigma}_{\theta}^{o(j)}, \bar{\tau}_{r \theta}^{o(j)}$ and $\bar{v}_{r}^{o(j)}, \bar{v}_{\theta}^{o(j)}$ for a reinforcing cylinder that correspond to the thermoelastic displacement potentials at each approximation. The found stresses and displacements for a drawing die and reinforcing cylinder will not satisfy boundary conditions (9), (10), respectively. At each approximation, it is necessary to find the second stress strain state: $\overline{=} d(j)=d(j)=d(j)=d(j)=d(j)$
$\sigma_{r}, \sigma_{\theta}, \tau_{r \theta}, v_{r}, v_{\theta}$ for a drawing die, and $\begin{gathered}\bar{\sigma}^{\prime}(j)=o(j)=o d(j)=o(j)=o(j) \\ \sigma_{r}, \sigma_{\theta}, \tau_{r \theta}, v_{r}, v_{\theta}\end{gathered}$ for a reinforcing cylinder so that the boundary conditions (9), (11) be fulfilled.

Consequently, for determining the second stress-strain state at a zero approximation, for a drawing die and reinforcing cylinder we have the following boundary conditions:

$$
\begin{array}{ll}
\text { for } r=R_{0} & \bar{\sigma}_{r}=d(o)=-p-\bar{\sigma}_{\theta}^{d(o)}, \bar{\tau}_{r \theta}^{d(o)}=-f p-\bar{\tau}_{r \theta}^{d(o)} \\
\text { for } r=R & \bar{\sigma}_{r}^{o(o)}-i \bar{\tau}_{r \theta}^{=o(o)}=\bar{\sigma}_{r}^{d(o)}-i \overline{\tau_{r \theta}}+f_{1}(\theta)
\end{array}
$$




$$
\begin{aligned}
& \begin{array}{l}
=o(o) \\
v_{r}-i v_{\theta}(o)
\end{array}=v_{r} v^{d(o)}-i v_{\theta}^{d(o)}-g^{(0)}(\theta)+f_{2}(\theta) \\
& \text { for } r=R_{1} \quad \overline{\bar{\sigma}}_{r}^{o(o)}=-\bar{\sigma}_{\theta}^{o(o)}, \bar{\tau}_{r \theta}^{o(o)}=-\bar{\tau}_{r \theta}^{o(o)} \\
& \text { for } y_{1}=0,\left|x_{1}\right| \leq l_{1} \quad \bar{\sigma}_{y_{1}}^{d(o)}=-\bar{\sigma}_{y_{1}}^{d(o)}, \bar{\tau}_{x_{1} y_{1}}^{d(o)}=-i \bar{\tau}_{x_{1} y_{1}}^{d(o)} \text {, }
\end{aligned}
$$

where $f_{1}(\theta)=\bar{\sigma}_{r}^{d(o)}-i \bar{\tau}_{r \theta}^{d(o)}-\left(\bar{\tau}_{r}^{o(o)}-i \bar{\tau}_{r \theta}^{o(o)}\right), \quad f_{2}(\theta)=\bar{v}_{r}^{d(o)}-i \bar{v}_{\theta}^{d(o)}-\left(\bar{v}_{r}^{o(o)}-i \bar{v}_{\theta}^{o(o)}\right)$.

We can write the boundary conditions of problem (16)-(17) by means of the Kolosov Muskheleshvili formulae [7] in the form of a boundary value problem for finding two pairs of complex potentials: $\Phi^{(0)}(z), \Psi^{(0)}(z)$ for the drawing die, $\Phi_{o}^{(0)}(z), \Psi_{o}^{(0)}(z)$ for the reinforcing cylinder.

We will seek the complex potentials in the form [7,10]

$$
\begin{aligned}
& \Phi^{0}(z)=\Phi_{1}^{(0)}(z)+\Phi_{2}^{(0)}(z)+\Phi_{3}^{(0)}(z), \Psi^{0}(z)=\Psi_{1}^{(0)}(z)+\Psi_{2}^{(0)}(z)+\Psi_{3}^{(0)}(z), \\
& \Phi_{1}^{(0)}(z)=\sum_{k=-\infty}^{\infty} d_{k} z^{k}, \quad \Psi_{1}^{(0)}(z)=\sum_{k=-\infty}^{\infty} c_{k} z^{k} \\
& \Phi_{2}^{(0)}(z)=\frac{1}{2 \pi} \int_{-l_{1}}^{l_{1}} \frac{g_{1}^{(0)}(t) d t}{t-z_{1}}, \quad \Psi_{2}^{(0)}(z)=\frac{1}{2 \pi} e^{-2 i \alpha_{1}} \int_{-l_{1}}^{l_{1}}\left[\frac{\overline{g_{1}^{(0)}(t)}}{t-z_{1}}-\frac{\bar{T}_{1} e^{i \alpha_{1}}}{\left(t-z_{1}\right)^{2}} g_{1}^{(0)}(t)\right] d t \\
& \Phi_{3}^{(0)}(z)=\frac{1}{2 \pi} \int_{-l_{1}}^{l_{1}}\left[-\frac{1}{z}-\frac{\overline{T_{1}}}{1-z \bar{T}} e^{i \alpha_{1}} g_{1}^{(0)}(t)+\overline{g_{1}^{(0)}(t)} e^{-i \alpha_{1}} \frac{1-T_{1} \bar{T}_{1}}{\overline{T_{1}}\left(1-z \overline{T_{1}}\right)^{2}}\right] d t \\
& \Psi_{3}^{(0)}(z)=\frac{1}{2 \pi} \int_{-l_{1}}^{l_{1}}\left\{g_{1}^{(0)}(t) e^{i \alpha_{1}}\left[\frac{1}{z T_{1}}-\frac{2}{z^{2}}-\frac{\overline{T_{1}}}{z\left(1-z \overline{T_{1}}\right)}+\frac{\overline{T_{1}}}{\left(1-z \overline{T_{1}}\right)^{2}}\right]+\right. \\
& \left.+\overline{g_{1}^{(0)}(t) e^{-i \alpha_{1}}}\left[\frac{1-T_{1} \bar{T}_{1}}{z \bar{T}_{1}\left(1-z \overline{T_{1}}\right)^{2}}-\frac{1}{1-z T_{1}}-\frac{2\left(1-T_{1} \bar{T}_{1}\right)}{\left(1-z \overline{T_{1}}\right)^{3}}\right]\right\} d t \\
& \Phi_{0}^{(0)}(z)=\sum_{k=-\infty}^{\infty} a_{k} z_{k}, \Psi^{(0)}(z)=\sum_{k=-\infty}^{\infty} b_{k} z_{k}
\end{aligned}
$$

Here $T_{1}=t e^{i \alpha_{1}}+z_{1}^{o}, \quad z_{1}=e^{-i \alpha_{1}}\left(z-z_{1}^{o}\right) ; g_{k}\left(x_{k}\right)$ are the desired functions, characterizing the displacement discontinuity across the crack line

$g_{k}^{(0)}(x)=\frac{2 G}{i(1+\kappa)} \frac{\partial}{\partial x}\left[u_{k}^{+}(x, 0)-u_{k}^{-}(x, 0)+i\left(v_{k}^{+}(x, 0)-v_{k}^{-}(x, 0)\right)\right]$,

$\kappa=3-4 \mu$, in the considered case $k=1$. 
Using (18)-(21) for finding complex potentials $\Phi_{1}^{(0)}(z), \Psi_{1}^{(0)}(z)$ and $\Phi_{0}^{(0)}(z), \Psi_{0}^{(0)}(z)$ we represent the boundary conditions in the form:

$$
\begin{aligned}
& \Phi_{1}^{(0)}\left(\tau_{0}\right)+\overline{\Phi_{1}^{(0)}\left(\tau_{0}\right)}-e^{2 i \theta}\left[\overline{\tau_{0}} \Phi_{1}^{(0)^{\prime}}\left(\tau_{1}\right)+\Psi_{1}^{(0)}(\tau)\right]=-p(1-i f)-\left(\bar{\sigma}_{r}^{d(0)}-i \bar{\tau}_{r \theta}\left({ }^{(0)}\right)\right. \\
& \Phi_{1}^{(0)}(\tau)+\overline{\Phi_{1}^{(0)}(\tau)}-e^{2 i \theta}\left[\bar{\tau} \Phi_{1}^{(0)}(\tau)+\Psi_{1}^{(0)}(\tau)\right]= \\
& =\Phi_{0}^{(0)}(\tau)+\overline{\Phi_{0}^{(0)}(\tau)}-e^{2 i \theta}\left[\bar{\tau} \Phi_{0}^{(0)^{\prime}}(\tau)+\Psi_{0}^{(0)}(\tau)\right]-f_{1}(\theta)-\left(f_{3}-i f_{4}\right) \\
& r=\rho(\theta)=R_{0}+\delta(\theta) \kappa \overline{\Phi_{1}^{(0)}(\tau)}-\Phi_{1}^{(0)}(\tau)+e^{2 i \theta}\left[\bar{\tau} \Phi_{1}^{(0)}(\tau)+\Psi_{1}^{(0)}(\tau)\right]= \\
& =\frac{G}{G_{0}}\left\{\kappa \overline{\Phi_{0}^{(0)}(\tau)}-\Phi_{0}^{(0)}(\tau)+\left[\bar{\tau} \Phi_{0}^{(0)}(\tau)+\Psi_{0}^{(0)}(\tau)\right] e^{2 i \theta}\right\}+2 G g^{(0)^{\prime}}(\tau)-f_{2}(\theta)-\left(f_{5}-i f_{6}\right) \\
& f_{3}-i f_{4}=\Phi_{*}^{(0)}(\tau)+\overline{\Phi_{*}^{(0)}(\tau)}-e^{2 i \theta}\left[\bar{\tau} \Phi_{*}^{(0)^{\prime}}(\tau)+\Psi_{*}^{(0)}(\tau)\right] \\
& f_{5}-i f_{6}=æ \overline{\Phi_{*}^{(0)}(\tau)}-\Phi_{*}^{(0)}(\tau)+e^{2 i \theta}\left[\bar{\tau} \Phi_{*}^{(0)^{\prime}}(\tau)+\Psi_{*}^{(0)}(\tau)\right] \\
& \Phi_{*}^{(0)}(\tau)=\Phi_{2}^{(0)}(\tau)+\Phi_{3}^{(0)}(\tau) ; \quad \Psi_{*}^{(0)}(\tau)=\Psi_{2}^{(0)}(\tau)+\Psi_{3}^{(0)}(\tau) \\
& \tau_{1}=R_{0} \exp (i \theta), \tau=\operatorname{Rexp}(i \theta) .
\end{aligned}
$$

We denote the left-hand side of the boundary condition (24) by the function $\sigma-i \tau$, then we have

$$
\Phi_{0}^{(0)}(\tau)+\overline{\Phi_{0}^{(0)}(\tau)}-e^{2 i \theta}\left[\tau \Phi_{0}^{(0)}(\tau)+\Psi_{0}^{(0)}(\tau)\right]-f_{1}(\theta)-\left(f_{3}-i f_{4}\right)=\sigma-i \tau
$$

We assume that the function $\sigma-i \tau$, which is a self-balanced system of forces acting on the reinforcing cylinder as viewed from the drawing die, can be expanded on the circular contour $L(\tau=\operatorname{Rexp}(i \theta))$ in a complex Fourier series

$$
\sigma-i \tau=\sum_{k=-\infty}^{\infty} A_{k} e^{i k \theta}
$$

For determining the complex potentials $\Phi_{0}^{(0)}(z)$ and $\Psi_{0}^{(0)}(z)$ we have condition (26) on the contour $L$, and the condition

$$
\Phi_{0}^{(0)}\left(\tau_{1}\right)+\overline{\Phi_{0}\left(\tau_{1}\right)}-e^{2 i \theta}\left[\bar{\tau}_{1} \Phi_{0}^{(0)}{ }^{\prime}\left(\tau_{1}\right)+\Psi_{0}^{(0)}\left(\tau_{1}\right)\right]=-\left(\bar{\sigma}_{r}^{0(0)}-i \bar{\tau}_{r \theta}^{0(0)}\right)
$$

on the contour $L_{1}\left(\tau_{1}=R_{1} \exp (i \theta)\right)$. 
The functions $\Phi_{0}^{(0)}(z)$ and $\Psi_{0}^{(0)}(z)$ are analytical in the interior of the transverse cross section of the reinforcing cylinder $R \leq|z| \leq R_{1}$ and may be represented [7] by the series (21). We use the power series method [7] to find the coefficients $a_{k}, b_{k}$ of the potentials $\Phi_{0}^{(0)}(z)$ and $\Psi_{0}^{(0)}(z)$.

For determining the still unknown quantities $A_{k}$, we consider the solution of the problem for a drawing die $R_{0} \leq|z| \leq R$. After some transformations of the complex potentials, $\Phi_{0}^{(0)}(z)$ and $\Psi_{0}^{(0)}(z)$ permit representing the boundary conditions for the functions $\Phi_{1}^{(0)}(z)$ and $\Psi_{1}^{(0)}(z)$ in the form (23) and

$$
\begin{aligned}
& \Phi_{0}^{(0)}(\tau)+\overline{\Phi_{1}^{(0)}(\tau)}-e^{2 i \theta}\left[\bar{\tau} \Phi_{1}^{(0)^{\prime}}(\tau)+\Psi_{1}^{(0)}(\tau)\right]=\sum_{k=-\infty}^{\infty} A_{k} e^{i k \theta} \\
& \kappa \overline{\Phi_{1}^{(0)}(\tau)}-\Phi_{1}^{(0)}(\tau)+\left[\bar{\tau} \Phi_{1}^{(0)^{\prime}}(\tau)+\Psi_{1}^{(0)}(\tau)\right] e^{2 i \theta}= \\
& =\sum_{k=-\infty}^{\infty} A_{k}^{*} e^{i k \theta}+2 G g^{(0)^{\prime}}(\tau)-f_{2}(\theta)-\left(f_{5}-i f_{6}\right) \\
& A_{-k}^{*}=\frac{G}{G_{0}}\left[\kappa_{0} \bar{a}_{k} R^{k}-a_{-k} R^{-k}(1+k)+b_{-k-2} R^{-k-2}\right] \\
& A_{k}^{*}=\frac{G}{G_{0}}\left[\kappa_{0} \bar{a}_{-k} R^{-k}+(k-1) a_{k} R^{k}+b_{k-2} R^{k-2}\right] .
\end{aligned}
$$

For the functions $\bar{\sigma}_{r}^{d(0)}-i \bar{\tau}_{r \theta}^{d(0)}, g^{\prime}(\tau), f_{2}(\theta),\left(f_{3}-i f_{4}\right),\left(f_{5}-i f_{6}\right)$ we will assume that they can be expanded in Fourier series

$$
\begin{aligned}
& -\left(\bar{\sigma}_{r}^{d(0)}-i \bar{\tau}_{r \theta}^{d(0)}\right)=\sum_{k=-\infty}^{\infty} A_{k}^{\prime} e^{i k \theta}, g^{(0)^{\prime}}(\tau)=\sum_{k=-\infty}^{\infty} A_{k}^{t} e^{i k \theta}, \\
& -\left(f_{5}-i f_{6}\right)=\sum_{k=-\infty}^{\infty} D_{k} e^{i k \theta},\left(f_{3}-i f_{4}\right)=\sum_{k=-\infty}^{\infty} B_{k} e^{i k \theta}, f_{2}(\theta)=\sum_{k=-\infty}^{\infty} A^{\prime \prime}{ }_{k} e^{i k \theta} .
\end{aligned}
$$

Here, the coefficients $D_{k}$ and $B_{k}$ depend on the desired function $g_{1}^{(0)}(t)$ and are determined by residue theory.

The boundary conditions (23), (29) are used to determine the coefficients $d_{k}, c_{k}$ and the boundary condition (30) is used to determine the quantities $D_{k}$. As a result, we find:

$$
d_{0}=\frac{A_{0} R^{2}-\left(A_{0}^{\prime}-p(1-i f)\right) R_{0}^{2}}{2\left(R^{2}-R_{0}^{2}\right)}, \quad d_{1}=\frac{\bar{A}_{1}^{\prime} R_{0}}{1+\kappa}, c_{-1}=-\kappa \frac{A_{1}^{\prime} R_{0}}{1+\kappa}
$$




$$
\begin{aligned}
& d_{1}=\frac{\bar{M}_{-1}}{R^{4}-R_{0}^{4}}-\frac{2 A_{1}^{\prime} R_{0}}{(1+\kappa)\left(R^{2}+R_{0}^{2}\right)}, \\
& d_{k}=\frac{(1+k)\left(R^{2}-R_{0}^{2}\right) M_{k}-\bar{M}_{-k}\left(R^{-2 k+2}-R_{0}^{-2 k+2}\right)}{\left(1-k^{2}\right)\left(R^{2}-R_{0}^{2}\right)^{2}-\left(R^{2 k+2}-R_{0}^{2 k+2}\right)\left(R^{-2 k+2}-R_{0}^{-2 k+2}\right)} \quad(k= \pm 2, \pm 3, \ldots), \\
& c_{-2} R_{0}^{-2}=2 d_{0}-\left(A_{0}^{\prime}-p(1-i f)\right), \\
& c_{k-2} R^{k-2}=(1-k) d_{k} R^{k}+\bar{d}_{-k} R^{-k}-A_{k}, \\
& M_{k}=A_{k} R^{-k+2}-A_{k}^{\prime} R_{0}^{-k+2}, \\
& (1+\kappa) \bar{d}_{0}=A_{0}+A_{0}^{*}+2 G A_{0}^{t}-A_{0}^{\prime \prime}+D_{0}, \\
& (1+\kappa) \bar{d}_{k} R_{k}=A_{-k}+A_{-k}^{*}+2 G A_{-k}^{t}-A_{-k}^{\prime \prime}+D_{-k}, \\
& (1+\kappa) \bar{d}_{-k} R^{-k}=A_{k}+A_{k}^{*}+2 G A_{k}^{t}-A_{k}^{\prime \prime}+D_{k} .
\end{aligned}
$$

The right-hand sides of the formulas for determining the coefficients $a_{k}, b_{k}, d_{k}, c_{k}$, and $A_{k}$ contain the coefficients of expansions of the allowance function $g^{(0)}(\theta)$ and also the integrals of the desired function $g_{1}^{(0)}(t)$.

Satisfying by the functions (18) the boundary condition (17) on the crack faces, we obtain a complex singular integral equation with respect to the unknown function $g_{1}^{(0)}(x)$

$$
\begin{aligned}
& \int_{-l_{1}}^{l_{1}}\left[R_{11}\left(t, x_{1}\right) g_{1}^{(0)}(t)+S_{11}\left(t, x_{1}\right) \overline{g_{1}^{(0)}(t)}\right] d t=\pi f_{0}\left(x_{1}\right),\left|x_{1}\right| \leq l_{1} \\
& f_{0}\left(x_{1}\right)=-\left[\Phi_{1}^{(0)}\left(x_{1}\right)+\overline{\Phi_{1}^{(0)}\left(x_{1}\right)}+x_{1} \overline{\Phi_{1}^{(0)}\left(x_{1}\right)}+\overline{\Psi_{1}^{(0)}\left(x_{1}\right)}\right]-\left(\bar{\sigma}_{y_{1}}^{d(0)}-i \bar{\tau}_{x_{1} y_{1}}^{d(0)}\right) .
\end{aligned}
$$

Here, the variables $x_{1}, t, l_{1}, z_{1}^{0}$ are dimensionless quantities referred to $R_{0}, R_{n k}, S_{n k}(n=k=1)$ are determined [10] by the formulae

$$
\begin{aligned}
& R_{n k}=\frac{e^{i \alpha_{k}}}{2}\left\{\frac{1}{T_{k}-X_{n}}+\frac{e^{-2 i \alpha_{n}}}{\bar{T}_{k}-\bar{X}_{n}}+\frac{1}{X_{n}\left(X_{n} \bar{T}_{k}-1\right)}+\right. \\
& \left.+\frac{1-T_{k} \bar{T}_{k}}{T_{k}\left(1-\bar{X}_{n} T_{k}\right)^{2}}+\frac{\left(T_{k} \bar{T}_{k}-1\right)\left(2 X_{n} T_{k} \bar{X}_{n}^{2}-3 \bar{X}_{n} T_{k}+1\right)+T_{k} \bar{X}_{n}\left(1-\bar{X}_{n} T_{k}\right)^{2}}{T_{k} \bar{X}_{n}^{2}\left(T_{k} \bar{X}_{n}-1\right)^{3}} e^{-2 i \alpha_{n}}\right\} \\
& S_{n k}(t, x)=\frac{e^{-i \alpha_{k}}}{2}\left\{\frac{1}{\bar{T}_{k}-\bar{X}_{n}}-\frac{T_{k}-X_{n}}{\left(\bar{T}_{k}-\bar{X}_{n}\right)^{2}} e^{-2 i \alpha_{n}}+\frac{1}{\bar{X}_{n}\left(T_{k} \bar{X}_{n}-1\right)^{+}}+\right.
\end{aligned}
$$




$$
\begin{aligned}
& +\frac{1-T_{k} \bar{T}_{k}}{\bar{T}_{k}\left(1-X_{n} \bar{T}_{k}\right)^{2}}+e^{-2 i \alpha_{n}}\left\langle\frac{1}{\bar{T}_{k} \bar{X}_{n}^{2}}+\frac{X_{n} \bar{X}_{n}\left(1-2 \bar{X}_{n} T_{k}\right)+3 \bar{X}_{n} T_{k}-2}{\bar{X}_{n}^{3}\left(1-\bar{X}_{n} T_{k}\right)^{2}}\right\rangle \\
& T_{k}=t e^{i \alpha_{k}}+z_{k}^{0} ; \quad z_{k}=e^{-i \alpha_{k}}\left(z-z_{k}^{0}\right) ; \quad X_{n}=x e^{i \alpha_{n}}+z_{n}^{0}
\end{aligned}
$$

For the inner crack, to the singular integral equation we should add additional equality expressing the displacement uniqueness condition in tracing the crack contour

$$
\int_{-l_{1}}^{l_{1}} g_{1}^{(0)}(t) d t=0
$$

Under the additional condition (33), the singular integral equation (32) by means of algebraization procedure (see the Appendix in [11]) is reduced to the system of $M$ algebraic equations for determining $M$ unknowns $g_{1}^{(0)}\left(t_{m}\right)(m=1,2, \ldots, M)$ :

$$
\begin{aligned}
& \frac{1}{M} \sum_{m=1}^{M} l_{1}\left[g_{1}^{(0)}\left(t_{m}\right) R_{11}\left(l_{1} t_{m}, l_{1} x_{r}\right)+\overline{g_{1}^{(0)}\left(t_{m}\right)} S_{11}\left(l_{1} t_{m}, l_{1} x_{r}\right)\right]=f_{0}\left(x_{r}\right) \quad(r=1,2, \ldots, M-1) \\
& \sum_{m=1}^{M} g_{1}^{(0)}\left(t_{m}\right)=0, \\
& t_{m}=\cos \frac{2 m-1}{2 M} \pi \quad(m=1,2, \ldots, M), x_{r}=\cos \frac{\pi r}{M} \quad(r=1,2, \ldots, M-1) .
\end{aligned}
$$

If in (34) we pass to the complex conjugate values, we will get $M$ additional algebraic equations. The obtained systems of equations with respect to $a_{k}, b_{k}, d_{k}, c_{k}, A_{k}, g_{1}^{(0)}\left(t_{m}\right)(m=1,2, \ldots, M)$ permit for a given allowance $g(\theta)$ to find the stress-strain state of a drawing die and reinforcing cylinder in the presence of a crack in the drawing die at a zero approximation. In the stated optimal design problem, the coefficients $A_{k}^{t}=\alpha_{k}+i \beta_{k}(k=0, \pm 1, \pm 2, \ldots)$ are to be determined. Consequently, the obtained united algebraic system is not still closed. For the stress intensity factors near the crack tips at a zero approximation we have:

$K_{I}^{(0)}-i K_{I I}^{(0)}=\sqrt{\pi l_{1}} \sum_{m=1}^{M}(-1)^{m} g_{1}^{(0)}\left(t_{m}\right) \cot \frac{2 m-1}{4 M} \pi \quad$ near the right vertex

$K_{I}^{(0)}-i K_{I I}^{(0)}=\sqrt{\pi t_{1}} \sum_{m=1}^{M}(-1)^{m+M} g_{1}^{(0)}\left(t_{m}\right) \tan \frac{2 m-1}{4 M} \pi \quad$ near the left vertex

For constructing the missing equations, we require the minimization of maximal value of the stress intensity factor

$K_{p \max }^{(0)} \rightarrow \min$ 
with regard to restrictions connected with carrying capacity, heat stability of a drawing die, unavailability of plastic deformations, and also the fact that

$K_{p \max }^{(0)} \leq K_{t h}$,

where $K_{t h}$ is the characteristic of the threshold value of the drawing die material fracture toughness which is determined experimentally.

At a zero approximation, the optimization problem is reduced to the definition of the coefficients (the control parameters) of the expansion of the allowance function $g^{(0)}(\theta)$ in the Fourier series. The quantities $g_{1}^{(0)}\left(t_{m}\right)$ linearly depend on the coefficients $A_{0}^{t(0)}$ of the Fourier series of the allowance function $g^{(0)}(\theta)$. Consequently, the quantity of the stress intensity coefficient (35) (the objective function) also linearly depends on the control parameters (control variables). Thus, using the minimax criterion, at a zero approximation, the considered problem is reduced to a linear programming problem.

Numerical calculations are performed by the simplex algorithm. In expansion of the allowance function $g^{(0)}(\theta)$ we were confined to seven terms. The calculations were conducted in conformity to the form of the drawing die $N_{0} 13$ [12]: $R_{1}=65 \mathrm{~mm}$; $2 R=29,5 \mathrm{~mm} ; 2 R_{0}=5,7 \mathrm{~mm}$. The mechanical characteristics of the drawing die material (hard alloy BK6;) and holders (mean carbon steel) were accepted to be equal to $E=6,28 \cdot 10^{5} \mathrm{MPa} ; v=0,22$ and $E_{0}=2,06 \cdot 10^{5} \mathrm{MPa} ; v_{0}=0,28$. The internal pressure $p$ changed within 391-1960 MPa.

After defining desired quantities of the zero approximation, we can go on to construct the solution of the problem at a first approximation. The functions $N_{0}$ and $T_{0}$ are determined on the base of the obtained solution for $r=R_{0}$. The boundary conditions (11) may be written in the form of a boundary value problem for finding complex potentials $\Phi^{(1)}(z), \Psi^{(1)}(z)$ and $\Phi_{0}^{(1)}(z), \Psi_{0}^{(1)}(z)$, which are sought in the form similar to (18)-(23) with obvious changes. The further course of the solution is the same as at a zero approximation. The obtained singular integral equation with respect to $g_{1}^{(1)}(t), \overline{g_{1}^{(1)}(t)}$ under additional condition of type (33) by means of the algebraization method is reduced to the system of $M$ algebraic equations for determining $M$ unknowns $g_{1}^{(1)}\left(t_{m}\right)(m=1,2, \ldots, M)$. The desired coefficients $a_{k}^{(1)}, b_{k}^{(1)}, d_{k}^{(1)}, c_{k}^{(1)}, A_{k}^{(1)}$ are contained in the right-hand side of this system.

In the stated optimal design problem, the coefficients $A_{k}^{t(1)}=\alpha_{k}^{(1)}+i \beta_{k}^{(1)}$ $(k=0, \pm 1, \pm 2, \ldots)$ should be defined. Consequently, the obtained united algebraic system is still closed. For stress intensity coefficients in the vicinity of the crack tips, at a first approximation we have:

$K_{I}^{(1)}-i K_{I I}^{(1)}=\sqrt{\pi t_{1}} \sum_{m=1}^{M}(-1)^{m} g_{1}^{(1)}\left(t_{m}\right) \cot \frac{2 m-1}{4 M} \pi \quad$ near the right vertex 
$K_{I}^{(1)}-i K_{I I}^{(1)}=\sqrt{\pi d_{1}} \sum_{m=1}^{M}(-1)^{m+M} g_{1}^{(1)}\left(t_{m}\right) \tan \frac{2 m-1}{4 M} \pi \quad$ near the left vertex

For constructing the missing equations, we use the minimax criterion

$K_{p \max }^{(1)} \rightarrow \min$

allowing for the above-mentioned restrictions.

The quantities $g_{1}^{(1)}\left(t_{m}\right)$ linearly depend on the coefficients $A_{k}^{t(1)}$ of the Fourier series of the allowance function $g^{(1)}(\theta)$.

Consequently, the quantity of stress intensity factor (37) (the objective function) also linearly depends on control parameters $A_{k}^{t(1)}$ (control variables). Thus, the optimization problem at a first approximation may also be reduced to a linear programming problem.

Numerical calculation was carried out by a symplex algorithm. The calculation results of the allowance function (the coefficients are given in $\mathrm{mm}$ ) are given in Table 1 for the case $\alpha_{1}=30^{\circ} ; l_{1} /\left(R-R_{0}\right)=0,1 ; z_{1}^{0}=\left(R_{0}+0,1\left(R-R_{0}\right)\right) e^{i \pi \theta / 12}$; $p=1200 \mathrm{MPa}$.

Table 1

The values of Fourier coefficients of optimal allowance

\begin{tabular}{|l|l|l|l|l|l|l|l|}
\hline$\alpha_{0}$ & $\alpha_{1}$ & $\alpha_{2}$ & $\alpha_{3}$ & $\alpha_{4}$ & $\alpha_{5}$ & $\alpha_{6}$ & $\alpha_{7}$ \\
\hline 0,1103 & 0,0792 & 0,0714 & 0,0642 & 0,0518 & 0,0489 & 0,0238 & 0,0157 \\
\hline & $\beta_{1}$ & $\beta_{2}$ & $\beta_{3}$ & $\beta_{4}$ & $\beta_{5}$ & $\beta_{6}$ & $\beta_{7}$ \\
\hline & 0,0718 & 0,0452 & 0,0423 & 0,376 & 0,0249 & 0,0202 & 0,0105 \\
\hline
\end{tabular}

If one of the crack ends the internal surface of the drawing die, then at each approximation, the equality (33) is replaced by an additional condition that expresses finiteness of stresses at the crack edge for $r=R_{0}$.

\section{The Case of an Arbitrary Number of Cracks}

Now we assume that in the elastic drawing die near the friction surface are $N$ rectilinear cracks of length $2 l_{k}(k=1,2, \ldots, N)$ (Fig. 1). We consider the optimal design problem, or more exactly, a problem of the definition of such an allowance function for the junction of the drawing die and the reinforcing cylinder that minimization of maximal value of the stress intensity factors near the crack tips.

In this case, the problem is solved by analogy with the problem for a single crack. The complex potentials $\Phi_{2}^{(j)}, \Psi_{2}^{(j)}$ and $\Phi_{3}^{(j)}, \Psi_{3}^{(j)}(j=0,1)$ are generalized to the 
case of arbitrary many cracks. By satisfying the boundary conditions on the crack edges, at each approximation we obtain the system of $N$ singular integral equations of the functions $g_{k}^{(j)}\left(x_{k}\right)(k=1,2, \ldots, N)$. At each approximation, to the system of singular integral equations for the inner cracks we should add the additional conditions

$$
\int_{-l_{k}}^{l_{k}} g_{k}^{(j)}(t) d t=0 \quad(j=0,1)
$$

At each approximation, under the above mentioned conditions by means of the algebraization process, the system of singular integral equations is reduced to the system of $N \times M$ algebraic equations for determining $N \times M$ unknowns $g_{k}^{(j)}\left(t_{m}\right)$ $(j=0,1 ; n=1,2, \ldots, N ; m=1,2, \ldots, M)$

$$
\begin{aligned}
& \frac{1}{M} \sum_{m=1}^{M} \sum_{k=1}^{N} l_{k}\left[g_{k}^{(j)}\left(t_{m}\right) R_{n k}\left(l_{k} t_{m}, l_{n} x_{r}\right)+\overline{g_{k}^{(j)}\left(t_{m}\right)} S_{n k}\left(l_{k} t_{m}, l_{n} x_{r}\right)\right]=f_{n}^{(j)}\left(x_{r}\right) \\
& \sum_{m=1}^{M} g_{n}^{(j)}\left(t_{m}\right)=0 .
\end{aligned}
$$

Construction of the missing equations is realized at each approximation similar to the case for one crack. For the stress intensity factors in the vicinity of the crack end at each approximation we get

$$
\begin{array}{ll}
K_{I n}^{(1)}-i K_{I I n}^{(1)}=\sqrt{\pi l_{n}} \sum_{m=1}^{M}(-1)^{m} g_{n}^{(1)}\left(t_{m}\right) \cot \frac{2 m-1}{4 M} \pi & \text { near the right vertex } \\
K_{I n}^{(j)}-i K_{I n}^{(j)}=\sqrt{\pi l_{n}} \sum_{m=1}^{M}(-1)^{m+M} g_{n}^{(j)}\left(t_{m}\right) \tan \frac{2 m-1}{4 M} \pi & \text { near the left vertex } \\
(j=0,1 ; n=1,2, \ldots, N ; m=1,2, \ldots, M) &
\end{array}
$$

Using the minimax criterion, in the case of an arbitrary number of cracks, the considered problem is reduced to a linear programming problem allowing for the mentioned restrictions.

The numerical calculation was carried out with a symplex algorithm for the same form of the drawing die. It is assumed that the drawing die is provided with three cracks:

$$
\begin{aligned}
& \alpha_{1}=45^{0}, l_{1} /\left(R-R_{0}\right)=0,05, z_{1}^{0}=\left(R_{0}+0,1\left(R-R_{0}\right)\right) e^{i \pi \theta / 18} \\
& \alpha_{2}=30^{0}, l_{2} /\left(R-R_{0}\right)=0,10, z_{2}^{0}=\left(R_{0}+0,15\left(R-R_{0}\right)\right) e^{i \pi \theta / 12} \\
& \alpha_{3}=15^{0}, l_{3} /\left(R-R_{0}\right)=0,075, z_{3}^{0}=\left(R_{0}+0,05\left(R-R_{0}\right)\right) e^{i \pi \theta / 10}
\end{aligned}
$$

The results of calculations of the allowance function (the coefficients are given in $\mathrm{mm})$ are cited in Table 2 . 
Table 2

The values of Fourier coefficients of optimal interference for the case of three cracks

\begin{tabular}{|l|l|l|l|l|l|l|l|}
\hline$\alpha_{0}$ & $\alpha_{1}$ & $\alpha_{2}$ & $\alpha_{3}$ & $\alpha_{4}$ & $\alpha_{5}$ & $\alpha_{6}$ & $\alpha_{7}$ \\
\hline 0,1291 & 0,0874 & 0,0719 & 0,0648 & 0,0566 & 0,0493 & 0,0341 & 0,0207 \\
\hline & $\beta_{1}$ & $\beta_{2}$ & $\beta_{3}$ & $\beta_{4}$ & $\beta_{5}$ & $\beta_{6}$ & $\beta_{7}$ \\
\hline & 0,0754 & 0,0687 & 0,0546 & 0,0481 & 0,0360 & 0,0204 & 0,0127 \\
\hline
\end{tabular}

The optimal solution, i.e. the found coefficients $\alpha_{k}$ and $\beta_{k}$, promote an increase the carrying capacity of a drawing die (drawing tool).

\section{A Simplified Method for Solving the Inverse Problem}

In the case of several cracks, the amount of calculations increases. We consider a simplified method for solving the problem of the definition of the optimal negative allowance for the junction of the drawing die and the reinforcing cylinder. Expand the desired allowance function in the Fourier series with that amount of terms equal to the number of cracks tips. In the case of $N$ internal cracks in the drawing die, we will be restricted by $2 \mathrm{~N}$ coefficients of the allowance function expansion in the Fourier series. We require that at each approximation the stress intensity factors in the vicinity of cracks ends be equal zero. Adding $2 \mathrm{~N}$ complex linear algebraic equations to the main resolving equations (they were discussed above) we get a closed algebraic system for defining all the unknowns, including the coefficients $\alpha_{k}^{(j)}, \beta_{k}^{(j)}(j=0,1)$ of the expansion of the allowance function into the Fourier series.

We assume that $N_{1}$ cracks have one end of a part on the internal surface of the drawing die. Then, the number of the cracks vertices will equal $\left(2 N-N_{1}\right)$. In this case, when a part of the cracks is a surface crack, then in expansion of the desired allowance function in the Fourier series we will use $\left(2 N-N_{1}\right)$ coefficients.

Require that the stress intensity factors near vertices be equal zero. Adding $\left(2 N-N_{1}\right)$ linear algebraic equations to the main resolving equations, we get in this case a closed algebraic system of equations for determining all the unknowns. It is appropriate to apply the simplified method for solving a problem on the minimization of fracture parameters of a drawing die involving a great amount of cracks, when the use of the symplex method causes a great volume of calculations. For the numerical solution of the obtained system of equations, we use the Gauss method with a choice of the principal element. Thus, the suggested methods of the minimization of failure parameters complete each other. 


\section{Conclusions}

The main equations obtained in the paper allow for the given negative allowance by numerical calculations, with the help of the definition of the stress intensity factors, to predict the growth of cracks existing in the drawing die and to establish the admissible level of deficiency and the maximal values of operation loads providing a sufficient reliability margin. At the design stage, the solution of the optimal design problem of the definition of the negative allowance in the junction of the drawing die and the reinforcing cylinder permits finding the optimal geometric parameters of the drawing die and the reinforcing cylinder, which ensures an increase in the carrying capacity. It should be noted that the obtained results are applicable in the case of a brittle fracture.

\section{References}

[1] Reshetov D. N.: 'State and Tendency of Machine Parts Development', Vestnik Mashinostroyeniya, No. 10, pp. 11-15, 2000

[2] Perlin I. L., Ermanok M. Z.: 'Drawing Theory' Metallurgiya, Moscow, 1971

[3] Zolgharnein E., Mirsalimov V. M.: 'Nucleation of a Crack under Inner Compression of Cylindrical Bodies', Acta Polytechnica Hungarica, Vol. 9, No. 2, pp. 169-183, 2012

[4] Thomas T. R.: 'Rough Surfaces' Longman, London, 1982

[5] Aykut Ş.: 'Surface Roughness Prediction in Machining Castamide Material Using ANN', Acta Polytechnica Hungarica, Vol. 8, No. 2, pp. 21-32, 2011

[6] Rusinko A., Rusinko K.: 'Plasticity and Creep of Metals' Springer, Verlag Berlin Heidelberg, 2011

[7] Mushelishvili N. I.: 'Some Basic Problems of Mathematical Theory Elasticity’ Amsterdam, Kluwer, 1977

[8] Cherepanov G. P.: 'Mechanics of Brittle Fracture' Mc Graw Hill, New York, 1979

[9] Parkus H.: 'Instationare Warmes-Pannungen' Springer, Wien, 1959

[10] Panasyuk V. V., Savruk M. P., Datsyshyn A. P.: 'A General Method of Solution of Two-Dimensional Problems in the Theory of Cracks', Eng. Fract. Mech., No. 2, pp. 481-497, 1977

[11] Mirsalimov V. M.: 'Non-One-Dimensional Elastoplastic Problems' Nauka, Moscow, 1987

[12] Samoilov V. S., Eichmans E. F., Falkovsky V. A. and others.: 'MetalWorking Hard - Alloy Tool' Reference book, Mashinostroyeniya, Moscow, 1958 